itself, for the determination of the 40 unknown functions $\Gamma_{\mu \nu}^{a}$. The following brief statement will serve to show how I have endeavoured to fill in this gap. ${ }^{3}$

If the German capital $\mathfrak{S}$ be a scalar density that depends only on the functions $\Gamma_{\mu \nu}^{a}$, then Hamilton's principle

$$
\delta\left\{\int \oiint_{\delta} d \tau\right\}=0
$$

supplies us with 40 differential equations for the functions $\Gamma$, when we stipulate that during the variation the functions $\Gamma$ are to be treated as magnitudes independent of each other. Further we assume that $\mathfrak{H}$ depends only on the magnitudes $\gamma_{\mu \nu}$ and $\phi_{\mu \nu}$, and thus write

where we have

$$
\delta \mathscr{F}_{\mathcal{C}}=\mathfrak{g}^{\mu \nu} \delta \hat{\gamma} \gamma_{\mu \nu}+\mathfrak{f}^{\mu \nu} \delta \phi_{\mu \nu} \quad . \quad .
$$

$$
\left.\begin{array}{l}
\frac{\partial \tilde{S}}{\partial \gamma_{\mu \nu}}=\mathfrak{g}^{\mu \nu} \\
\frac{\partial \mathcal{S}_{\tilde{C}}}{\partial \phi_{\mu \nu}}=\mathfrak{f}^{\mu \nu}
\end{array}\right\}
$$

At this point it should be noticed that in the theory developed here, the small German letters respectively represent the contravariant density $\left(\mathrm{g}^{\mu \nu}\right)$ of the metrical tensor, and the contravariant tensor density $\left(f^{\mu \nu}\right)$ of the electromagnetic field. Thus in a well-known manner is given the transition from tensor densities (expressed by German letters) to contravariant and covariant tensors (expressed by the corresponding italic letters), and a metric is introduced which rests exclusively on the affine relation.

By performing the variation we obtain after some amount of calculation

$$
\Gamma_{\mu \nu}^{a}=\frac{1}{2} g^{\alpha \beta}\left(\frac{\partial g_{\mu \beta}}{\partial x_{\nu}}+\frac{\partial g_{\nu \beta}}{\partial x_{\mu}}-\frac{\partial g_{\mu \nu}}{\partial x_{\beta}}\right)-\frac{1}{2} g_{\mu \nu} i^{a}+\frac{1}{6} \delta_{\mu}^{a} i_{\nu}+\frac{1}{6} \delta_{\nu}^{\alpha} i_{\mu}
$$

where

$$
\frac{\partial \tilde{T}^{\mu \nu}}{\partial x_{\nu}}=\dot{i}^{\mu} . \quad . \quad . \quad . \quad .
$$

Equation (8) shows that our extension of the theory, which appears to be so general, leads to a structure of the affine relation that does not deviate more strongly from that of the geometry of Riemann than is required by the actual structure of the physical field.

We now obtain the field equations in the following manner. From (3) and (4) we first derive the relations

$$
\gamma_{\mu \nu}=-\frac{\partial \Gamma_{\mu \nu}^{\alpha}}{\partial x_{\alpha}}+\Gamma_{\mu \beta}^{\alpha} \Gamma_{\nu \alpha}^{\beta}+\frac{1}{2}\left(\frac{\partial \Gamma_{\mu \alpha}^{\alpha}}{\partial x_{\nu}}+\frac{\partial \Gamma_{\nu \alpha}^{\alpha}}{\partial x_{\mu}}\right)-\Gamma_{\mu \nu}^{\alpha} \Gamma_{\alpha \beta}^{\beta} \text { (10) }
$$
${ }^{3}$ Herr Droste of Leyden hit upon the same idea independently of the
present writer.

$$
\phi_{\mu \nu}=\frac{1}{2}\left(\frac{\partial \Gamma_{\mu \alpha}^{\alpha}}{\partial x_{\nu}}-\frac{\partial \Gamma_{i \alpha}^{\alpha}}{\partial x_{\mu}}\right) . \quad . \quad .
$$

In these equations the $\Gamma_{\mu \nu}^{\alpha}$ on the right-hand side are to be expressed by means of (8) in terms of the $\mathfrak{g}^{\mu \nu}$ and $\mathfrak{f}^{\mu \nu}$. Moreover, if $\mathfrak{S}$ is known, then on the basis of $(7) \gamma_{\mu \nu}$ and $\phi_{\mu \nu}$, i.e. the left-hand sides of (10) and (II), can also be expressed in terms of $\mathrm{g}^{\mu \nu}$ and $f_{\mu \nu}$. This latter calculation can be simplified by means of the following artifice. Equation (6) is equivalent to the statement that

$$
\delta \mathfrak{S}^{*}=\gamma_{\mu \nu} \delta \mathrm{g}^{\mu \nu}+\phi_{\mu \nu} \delta \tilde{f}^{\mu \nu}
$$

is also a complete differential, so that if $\mathfrak{S}^{*}$ is an unknown function of the $g^{\mu \nu}$ and $f^{\mu \nu}$, the following relations will hold:

$$
\left.\begin{array}{rl}
\gamma_{\mu \nu} & =\frac{\partial \mathscr{S}^{*}}{\partial \mathfrak{g}^{\mu \nu}} \\
\phi_{\mu \nu} & =\frac{\partial \mathcal{S}^{*}}{\partial \mathfrak{f}^{\mu \nu}}
\end{array}\right\}
$$

We now have only to assume $\mathfrak{S}^{*}$. The simplest possibility is obviously

$$
\mathfrak{S}^{*}=-\frac{\beta}{2} f_{\mu \nu} \mathfrak{f}^{\mu \nu}
$$

In this connexion it is interesting that this function does not consist of several summation terms which are logically independent of each other, as was the case with the theories hitherto proposed.

In this way we arrive at the field equations

$$
R_{\mu \nu}=-\kappa\left[\left(\frac{1}{4} g_{\mu \nu} f_{\sigma \tau} f^{\sigma \tau}-f_{\mu \sigma} f_{\nu}^{\sigma}\right)+\gamma f_{\mu} f_{\nu}\right] .
$$

whereby $R_{\mu \nu}$ is the Riemann tensor of curvature. $\kappa$ and $\gamma$ are constants, $f_{\mu}$ is the electromagnetic potential, which is connected with the field strength by the relation

$$
f_{\mu \nu}=\frac{\partial f_{\mu}}{\partial x_{\nu}}-\frac{\partial f_{\nu}}{\partial x_{\mu}}
$$

and with the electrical current density by the relation

$$
\dot{i}^{\mu}=-\gamma \mathfrak{g}^{\mu \sigma} f_{\sigma}
$$

In order that these equations may be in accord with experience, the constant $\gamma$ must be practically indefinitely small, for otherwise no fields would be possible without noticeable electrical densities.

The theory supplies us, in a natural manner, with the hitherto known laws of the gravitational field and of the electromagnetic field, as well as with a connexion as regards their nature of the two kinds of field; but it brings us no enlightenment on the structure of electrons.

\title{
Further Determinations of the Constitution of the Elements by the Method of Accelerated Anode Rays. ${ }^{1}$
}

By Dr. F. W. Aston, F.R.S.

$\mathrm{BY}$ further ase of the method of accelerated anode rays, results have been obtained with a number of elements since the publication of the isotopes of copper (Nature, Aug. 4, p. x62). Details of the

${ }^{1}$ A paper read on September 18 before Section A of the British Association Meeting at Liverpool. method will be published later. Most of the following results were obtained by the use of fluorine compounds of the elements investigated.

The mass-spectrum of strontium shows one line only; at 88 . This was obtained in considerable intensity. If any other constituents exist they must be present

NO. 28 I 2 , VOL. II 2$]$ 
in very small quantities, so that it is practically certain that the chemical atomic weight $87^{\circ} 63$ at present in use is too low.

Cobalt also appears to be a simple element of massnumber 59, as was to be expected from its atomic weight, which has been determined with great care by a number of observers.

Scandium was successfully attacked by the use of material kindly supplied by Prof. Urbain, of Paris. The only line obtained was at 45 . It may be taken provisionally to be a simple element, but the effects are not strong enough to disprove the presence of small quantities of another constituent.

Manganese behaved surprisingly well, and yielded unequivocal results indicating that it is a simple element of mass-number 55. This result is in good agreement with the chemical atomic weight, and is particularly interesting, for 55 is a term in the numerical series $2,3,5,8$, I3-all of which had previously corresponded to gaps in the list of weights of known species of atoms.

Gallium fluoride made from a specimen of the hydrate kindly provided by Prof. Richards, of Harvard University, also gave satisfactory results. Gallium consists of two isotopes, 69 and $7 \mathrm{I}$. The intensity relation between the lines agrees much better with the atomic weight 69.72 recently published by Richards than that previously in use, $70 \cdot \mathrm{I}$.

Vanadium and chromium give single mass-lines at positions expected from their atomic weights 51 and 52 .

Titanium gives a strong line at 48 . On one of the spectra obtained there is a faint and doubtful indication of a line at 5o. Should this latter be confirmed it would tend to support Honigschmid's value $48 \cdot x$ for the atomic weight rather than the lower figure $47 \cdot 85$ more recently obtained by Baxter.

Silver in the form of the chloride worked unexpectedly well, and gave two nearly equally intense lines at го7, 109.
Yttrium gives a single strong line at 89 , another term of the numerical series already referred to, and completes the analysis of the first 39 elements.

A specimen of potassium hafnifluoride sent from Copenhagen by Dr. Hevesy wàs experimented with, but in no case were any lines visible in the region of the expected atomic weight of hafnium. This sample contained about 50 per cent. of zirconium, and an extremely faint effect at 90 shown here and on other plates taken with pure zirconium salts suggests this as the principai isotope of this element; but further work is necessary on this point.

Niobium, molybdenum, cadmium, barium, and lead have all been tried without any definite results, and it is feared that difficulties may arise in finding suitable compounds to use in the case of these and other elements not yet analysed. On the other hand, success with scandium and yttrium offers hope of obtaining the mass-spectra of all the rare-earth group.

The following is a list of the elements the composition of which has been first indicated by the use of accelerated anode rays. The mass-numbers were usually determined with reference to the lines of iron or iodine, and no outstanding divergence from the whole-number rule was observed.

\begin{tabular}{|c|c|c|c|c|}
\hline Element. & $\begin{array}{c}\text { Atomic } \\
\text { Number. }\end{array}$ & $\begin{array}{l}\text { Atomic } \\
\text { Weight. }\end{array}$ & $\begin{array}{l}\text { Minimum Number } \\
\text { of Isotopes. }\end{array}$ & $\begin{array}{l}\text { Mass-numbers in } \\
\text { Order of Intensity. }\end{array}$ \\
\hline $\mathrm{Sc}$ & $2 \mathrm{I}$ & $45^{\circ} \mathrm{x}$ & $\mathrm{x}$ & 45 \\
\hline $\mathrm{Ti}$ & 22 & $48 \cdot I$ & I & 48 \\
\hline V & 23 & $5^{\circ} \circ$ & I & $5^{x}$ \\
\hline $\mathrm{Cr}$ & 24 & $5^{\circ} \circ$ & I & $5^{2}$ \\
\hline Mn & 25 & $54^{\circ} 93$ & I & 55 \\
\hline Co & 27 & $58 \cdot 97$ & I & 59 \\
\hline $\mathrm{Cu}$ & 29 & $63 \cdot 57$ & 2 & 63,65 \\
\hline $\mathrm{Ga}$ & $3 I$ & $69^{\circ} 72$ & 2 & $69,7 \mathrm{I}$ \\
\hline $\mathrm{Ge}$ & $3^{2}$ & $72 \cdot 5$ & 3 & $74,72,70$ \\
\hline $\mathrm{Sr}$ & $3^{8}$ & $87 \cdot 63$ & I & 88 \\
\hline $\mathrm{Y}$ & 39 & $88 \cdot 9$ & $x$ & 89 \\
\hline $\mathrm{Ag}$ & 47 & 107.88 & 2 & 107,109 \\
\hline
\end{tabular}

\section{Obituary.}

Sir Henry Hubert Hayden, F.R.S.

A LL who knew Sir Henry Hayden well enough must A recall a passing thought, more than once definitely formulated, that some day his irrepressible keenness for exploring new and little-known lands would lead to accident. Those who had the inestimable privilege of knowing him with real intimacy know well that, if he had to choose a way of ending his career, it would be on a mountain side and in a fight against physical difficulties. He never revealed and probably never entertained but one fear-that the medical history of his family might repeat itself and render him unfit for further exploratory work. It is appropriate that he should be laid to rest near the foot of a great mountain, and appropriate too that it should be the mountain which he had just conquered, for as a mountaineer he was as efficient as he was daring.

To accomplish a difficult task in exploration was in itself his sufficient reward. Kindred spirits and but very few others knew of his accomplishments; for, without being reticent, he never looked to the "gallery": his photographs, maps, sketches, and collections were made readily available to specialists, but rarely, and only under pressure, were they turned into lantern slides. The end of one task was to him the beginning of the next: there never was an interval for popular demonstrations, and little even for rest.

Each geographical enterprise was invariably in a new field, and Hayden's geological work was just as varied - geotectonic problems in the Himalayas, economic mineral questions in various parts of India, pure palæontology, the application of geology to engineering problems, and the microscopic petrology of igneous rocks formed the subjects of his papers, each treated in turn with a thoroughness and sense of relativity that revealed a wide and precise acquaintance with literature, which was always surprising to those who were impressed by his restless physical activity in the field.

Since January 3, I895, when I met Hayden on his landing at Calcutta to join the Geological Survey of India, I have been in closer and perhaps more constant touch with him than most of his friends, and during those twenty-eight years I never heard from him an ungenerous remark about a colleague, never heard him grumble about the climate, at the work, or even at the inequalities of treatment that seem to be the

NO. 28 I 2 , VOL. I I 2 ] 